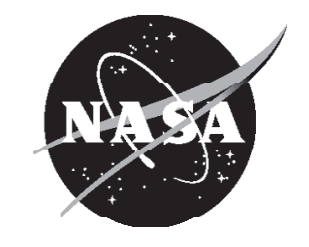

\title{
Estimation of the Unsteady Aerodynamic Load on Space Shuttle External Tank Protuberances From a Component Wind Tunnel Test
}

Jayanta Panda

Ohio Aerospace Institute, Brook Park, Ohio

Fred W. Martin

Johnson Space Center, Houston, Texas

Daniel L. Sutliff

Glenn Research Center, Cleveland, Ohio 


\section{NASA STI Program . . . in Profile}

Since its founding, NASA has been dedicated to the advancement of aeronautics and space science. The NASA Scientific and Technical Information (STI) program plays a key part in helping NASA maintain this important role.

The NASA STI Program operates under the auspices of the Agency Chief Information Officer. It collects, organizes, provides for archiving, and disseminates NASA's STI. The NASA STI program provides access to the NASA Aeronautics and Space Database and its public interface, the NASA Technical Reports Server, thus providing one of the largest collections of aeronautical and space science STI in the world. Results are published in both non-NASA channels and by NASA in the NASA STI Report Series, which includes the following report types:

- TECHNICAL PUBLICATION. Reports of completed research or a major significant phase of research that present the results of NASA programs and include extensive data or theoretical analysis. Includes compilations of significant scientific and technical data and information deemed to be of continuing reference value. NASA counterpart of peer-reviewed formal professional papers but has less stringent limitations on manuscript length and extent of graphic presentations.

- TECHNICAL MEMORANDUM. Scientific and technical findings that are preliminary or of specialized interest, e.g., quick release reports, working papers, and bibliographies that contain minimal annotation. Does not contain extensive analysis.

- CONTRACTOR REPORT. Scientific and technical findings by NASA-sponsored contractors and grantees.

- CONFERENCE PUBLICATION. Collected papers from scientific and technical conferences, symposia, seminars, or other meetings sponsored or cosponsored by NASA.

- SPECIAL PUBLICATION. Scientific, technical, or historical information from NASA programs, projects, and missions, often concerned with subjects having substantial public interest.

- TECHNICAL TRANSLATION. Englishlanguage translations of foreign scientific and technical material pertinent to NASA's mission.

Specialized services also include creating custom thesauri, building customized databases, organizing and publishing research results.

For more information about the NASA STI program, see the following:

- Access the NASA STI program home page at http://www.sti.nasa.gov

- E-mail your question via the Internet to help@ sti.nasa.gov

- Fax your question to the NASA STI Help Desk at 301-621-0134

- Telephone the NASA STI Help Desk at 301-621-0390

- Write to: NASA Center for AeroSpace Information (CASI) 7115 Standard Drive Hanover, MD 21076-1320 


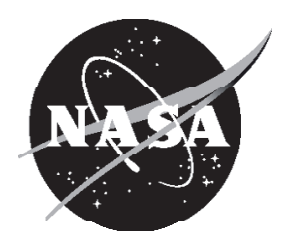

\section{Estimation of the Unsteady Aerodynamic Load on Space Shuttle External Tank Protuberances From a Component Wind Tunnel Test}

Jayanta Panda

Ohio Aerospace Institute, Brook Park, Ohio

Fred W. Martin

Johnson Space Center, Houston, Texas

Daniel L. Sutliff

Glenn Research Center, Cleveland, Ohio

Prepared for the

46th Aerospace Sciences Meeting and Exhibit

sponsored by the American Institute of Aeronautics and Astronautics

Reno, Nevada, January 7-10, 2008

National Aeronautics and

Space Administration

Glenn Research Center

Cleveland, Ohio 44135 


\section{Acknowledgments}

The first and the third author acknowledge support from the NASA Engineering Safety Center (NESC) in performing this work. The IS-23 test was conceived and conducted by a large group of engineers and support staff from several NASA centers, USA and Boeing. Dr. Jim Peters of the Space Shuttle Systems Engineering and Integration Office was the Principal Investigator for this test. Dr. Mark Seaford and Dr. Darren Reed's contributions, from the Marshall Space Flight Center, were invaluable in planning and conducting the test, along with the guidance obtained from Dr. John Edwards at the Langley Research Center. The contribution from the following Boeing employees was critical to the success of the test. Mr. Rick Burrows served as the Test Engineer, Mr. Lung Wong, as the Lead Aerodynamic Analyst, and Dr. Ian Fialho, as the Lead Dynamic Data Analyst. Mr. Pete Pozefsky and Mr. Louis Hall were instrumental in processing and presenting the dynamic data during the test. We would have never remained on schedule without the help of the Project Engineers, Mr. Mike Oelke and Mr. Darby Cooper. Finally, the NASA Glenn Research Center model, instrumentation, and wind tunnel staff were all essential with Mr. Scott Williamson deserving special recognition as the Facility Test Engineer.

Level of Review: This material has been technically reviewed by technical management.

Available from

NASA Center for Aerospace Information 7115 Standard Drive

Hanover, MD 21076-1320
National Technical Information Service 5285 Port Royal Road Springfield, VA 22161

Available electronically at http://gltrs.grc.nasa.gov 


\title{
Estimation of the Unsteady Aerodynamic Load on Space Shuttle External Tank Protuberances From a Component Wind Tunnel Test
}

\author{
Jayanta Panda \\ Ohio Aerospace Institute \\ Brook Park, Ohio 44142 \\ Fred W. Martin \\ National Aeronautics and Space Administration \\ Johnson Space Center \\ Houston, Texas 77058 \\ Daniel L. Sutliff \\ National Aeronautics and Space Administration \\ Glenn Research Center \\ Cleveland, Ohio 44135
}

\begin{abstract}
At the wake of the Columbia (STS-107) accident it was decided to remove the Protuberance Aerodynamic Load (PAL) Ramp that was originally intended to protect various protuberances outside of the Space Shuttle External Tank from high buffet load induced by cross-flows at transonic speed. In order to establish the buffet load without the PAL ramp, a wind tunnel test was conducted where segments of the protuberances were instrumented with dynamic pressure transducers; and power-spectra of sectional lift and drag forces at various span-wise locations between two adjacent support brackets were measured under different cross flow angles, Mach number and other conditions. Additionally, frequencydependent spatial correlations between the sectional forces were also established. The sectional forces were then adjusted by the correlation length to establish span-averaged spectra of normal and lateral forces that can be suitably "added" to various other unsteady forces encountered by the protuberance. This paper describes the methodology used for calculating the correlation-adjusted power spectrum of the buffet load. A second part of the paper describes wind-tunnel results on the difference in the buffet load on the protuberances with and without the PAL ramp. In general when the ramp height is the same as that of the protuberance height, such as that found on the liquid Oxygen part of the tank, the ramp is found to cause significant reduction of the unsteady aerodynamic load. However, on the liquid Hydrogen part of the tank, where the Oxygen feed-line is far larger in diameter than the height of the PAL ramp, little protection is found to be available to all but the Cable Tray.
\end{abstract}

\section{Introduction}

Following the Space Shuttle STS-114 mission, it was decided to remove the Protuberance Aerodynamic Load (PAL)
Ramp that was originally intended to protect various protuberances, mounted on the outside of the Space Shuttle External Tank, from aerodynamic buffet loads induced by cross-flows at transonic speeds. The Space Shuttle External Tank (ET) carries the cryogenic propellants for the Space Shuttle Main Engines (SSME) in two tanks, with the Liquid Oxygen (LOX) tank forming the forward ogive of the ET and the Liquid Hydrogen (LH2) tank forming the cylindrical section as shown in figures 1 and 2 . The two tanks are joined together by a corrugated Intertank structure and the entire exterior is covered in insulating foam that is primarily sized to prevent ice formation prior to launching the vehicle. In some areas, however, ascent heating dictates the insulation thickness.

Several critical sub-systems are routed around the tanks on the exterior as can be seen in figure 2. Two cable trays carry flight instrumentation wiring to the top of both tanks, and hot gaseous hydrogen (GH2) and oxygen (GO2) flow up to the top of their respective tanks through the two Pressurization Lines (press lines). The gaseous propellants are used to maintain flight pressure in the tanks as the liquid propellant is used by the engines. The LOX Feedline is the large pipe which exits the Intertank and runs aft along the LH2 tank just inboard of the press. lines.

During the development of the Space Shuttle, oil flow images obtained from small-scale $(0.4$ percent model) wind tunnel tests indicated that the LOX tank cable tray and press line protuberances would experience significant amounts of cross flow while the launch vehicle was passing through the transonic Mach range. Severe boundary layer separation patterns were noticed when the Solid Rocket Booster bow shock waves crossed the LOX tank just forward of the Intertank. The oil flow patterns indicated that up to $90^{\circ}$ cross flow could occur. Pressure data, from a larger scale test ( 2 and 3 percent) was used to determine that the local Mach number was in the transonic range. This led the design teams to be very concerned that the LOX tank cable tray may experience 
an aero-elastic instability during transonic flight, which would lead to a catastrophic failure of the cable tray and vehicle. Wind tunnel testing of the original cable tray shape, which was roughly twice as wide as the current cable tray, shown in figure 2, indicated that shock waves would form parallel to the long axis when the cable tray experienced transonic cross flow. An aero-elastic analysis, which assumed 1 percent damping for the cable tray structure, showed that the cable tray would experience an undamped oscillation leading to failure of the tray. Thus the design teams decided to protect the protuberances from the transonic cross flow with foam ramps. The LO2 Protuberance Air Load Ramp (PAL) and the LH2 PAL ramp can both be seen in figure 2 .

The aerodynamic cross flow, on the hydrogen tank, is a complicated function of flight Mach number, Angle-of-Attack, and location along the tank. In general, it is much smaller in magnitude than on the LO2 tank, never reaching the extreme $90^{\circ}$ flow angle seen there. The pre-flight analysis never conclusively indicated that the LH2 tank cable tray would experience catastrophic aero-elastic instability; however, a PAL ramp was added to the LH2 tank to minimize the effects of cross flow on the protuberances.

The PAL ramps remained a part of the ET design until after STS-114, which was the Return to Flight mission that followed the loss of Columbia on STS-107. During the stand down period, between the two missions, engineers at the Marshall Space Flight Center, working with their colleagues at the Langley Research Center and Lockheed-Martin, the ET contractor, proactively began work on characterizing the aeroelastic response of full scale cable trays in a transonic cross flow. They also ran structural modal tests that measured significant structural damping in the cable tray system due to the sliding joint between the trays which accommodates the contraction in the ET when the cryogenic propellants are loaded. During the launch of STS-114, a large piece of foam was shed from the LH2 PAL ramp, which fortunately passed safely underneath the Orbiter wing. The event, however, was significant enough to once again cause the Shuttle Program to stand down until the engineering could be completed to prove that the PAL Ramps were not necessary and could be removed from the design.

Computational Fluid Dynamic (CFD) codes were used to estimate the steady part of the aerodynamic forces and to establish the average aerodynamic environment. The steady aerodynamic forces and moments and the associated structural responses were also measured in many wind tunnel studies (ref. 1). However, the unsteady part of the aerodynamic forces was not measured. The current CFD codes have limitations in predicting this unsteady part. Therefore two other options were pursued: an engineering estimate based on analytical efforts, and direct wind tunnel tests. The semi-empirical analytical method proposed by Warner Dahm (ref. 2) and Phineas Woods (ref. 3) was used to create a Preliminary
Design Review (PDR) environment. The present paper is based on a component wind tunnel test (IS-23) where twodimensional scale models of the protuberances were tested in a transonic wind tunnel to determine unsteady sectional forces. A 50 percent scaled-model of the LOX tank protuberances that run outside the upper part of the ET, and a 25 percent scaledmodel of the protuberances on the LH2 portion of the ET were used. The wind-tunnel data was used to create an aerodynamic buffet environment for the Critical Design Review (CDR).

The purpose of the component wind tunnel test was to provide a conservative estimate of the unsteady buffet load experienced by the External Tank protuberances. The total unsteady load is made of the vibration transmitted from the tank skin through the brackets (base drive) and the unsteady aerodynamic load. The test was setup to estimate the last item. The buffet load estimated from this test was suitably "added" to the base drive model in structural analysis computer codes to predict the resultant structural response. Notably, the test articles were rigid with stiffness values different from that of the flight articles; therefore, little effort was made to measure structural response in this test. Additionally, the real flow over the protuberances is highly three-dimensional, while the component wind tunnel test simulated a two-dimensional environment such that a conservative estimate of the unsteady forces would be obtained. Also the wind tunnel test simulated only a very small part of the actual long protuberances with their multitude of complexities. Various scaling factors, derived from the Shuttle launch vehicle three-dimensional CFD results, were used to relate the wind tunnel data to the actual flight vehicle.

\section{Scope of This Paper}

The scope is narrowed down to two fundamental aspects. First: to describe the technique used to estimate an average spectrum of unsteady dynamic forces from a large number of unsteady surface pressure measurements. One set of data acquired during the test is used to describe this technique. The technique lies at the heart of the process to create the buffet load environment. However various details of enveloping the test data, additional scaling for different regions of the protuberances etc., are left out. Additionally, for the present paper the forces are non-dimensionalized to lift and drag coefficients and frequencies to Strouhal number to help future use of the data. The CDR environment described the forces in dimensional terms. The second part of this paper describes changes in unsteady lift and drag force coefficients experienced by the model scale protuberances due to the removal of the PAL ramp. Towards these two goals attempts are made to describe the influence of various fluid phenomena, such as the coherent vortex shedding, flow over rectangular and circular objects in the vicinity of solid wall etc. The present paper does not attempt to document the vast data base collected during the test. 


\section{Test Articles and Test Facility}

The original intention of the PAL ramp was to protect the ET protuberances from significant cross-flows. Various earlier flow-visualization images showed that during the transonic flight regime shock waves emanating from the SRB and Orbiter nose cone impinged upon the External Tank and created the potential for cross-flow velocity (ref. 1) in the transonic Mach range. When the PAL ramp is removed the protuberances were to face the cross flow once again. Extensive data mining from Computational Fluid Dynamics (CFD) analysis of the full stack vehicle at different flight Mach numbers, angle of attack, and sideslip angles was used for establishing the extent of this cross-flow and also for selection of the test articles and defining the test matrix. The data extracted provided local cross-flow angle, local Mach number, and local dynamic pressure. These provided guidance on setting tunnel Mach number, scaling parameters and cross-flow angles applicable to different segments of the protuberances.

The test was conducted in the 8- by 6-ft Supersonic Wind Tunnel of the NASA Glenn Research Center in Cleveland, Ohio. The testing began in February 2006 and ended in early April 2006. Various pre- and post-test engineering reports by USA/Boeing (refs. 4 to 6) provide a detail description of the test articles. The following description only covers the essential skeleton.

The sections of the protuberances that were simulated in the wind tunnel test are shown in figures 3 and 4 . There were two sections from two different parts of the ET: the top Liquid Oxygen (LO2) and the bottom liquid Hydrogen (LH2) parts. The test articles were mounted on a splitter plate that simulated the solid surface of the External Tank (fig. 3(a)). The actual flow on the protuberances is expected to be very threedimensional; while the wind tunnel simulated a twodimensional flow; the side-rails on the splitter plate helped create such a flow. The three-dimensional inboard-outboard curvature of the ET was simulated by providing a slight pitch to the Cable Tray (fig. 4(b)). So that the measured environment can be applied over different segments of the protuberance, with variable heights from the tank surface, the height and pitch angle of the Cable Tray were varied in the test. In addition to the Cable Tray configurations, other local variables such as LO2 feedline flange and bracket placement, PAL-on/ PAL-off, and different Ice Frost Ramp (IFR) geometries were also tested.

\section{Brief Description of LH2 Test Articles and Dynamic Pressure Instrumentation}

The 25 percent scaled LH2 model had four different articles: a liquid Oxygen (LOX) feedline, a gaseous Oxygen (GO2) pressurization line, a gaseous Hydrogen (GH2) pressurization line and a Cable Tray. Figure 3 shows a schematic of the articles and locations of some of the dynamic pressure transducers. The Ice/Frost ramps (IFR) were built over each attachment point of the protuberances on the tank. Their particular shapes correspond to that of the insulating foam molding used for eliminating condensation and ice formation on the supporting brackets for the protuberances. The test articles were long enough to place six IFRs over the model length. Each IFR was bolted to the splitter plate. Two different IFR geometries were tested. Figure 3 shows the old IFR shapes; a redesigned 'new' shape with smaller profile was also tested.

To simulate cross-flows in both outboard-inboard (positive) and inboard-outboard (negative) directions, test articles were subjected to both positive and negative cross flow velocities. The latter was obtained by translating GO2 and $\mathrm{GH} 2$ pressure lines and LOX feedline forward of the cable tray, which always remained stationary for both models. Simulating negative cross flow angles required mirror-image IFRs for proper geometry simulation.

A total of 86 high frequency absolute pressure transducers (Kulite) and 40 static pressure taps were installed on the LH2 test articles. The dynamic pressure transducers were bunched in groups of 4 to 16 transducers to measure sectional aerodynamic forces on each protuberance. Most of these instruments were placed in the middle instrumented segment of the protuberances. There were 48 Kulites installed on the Cable Tray at five different stations: stations 2, 3, 5, and 6 in figure 3(a) had 8 Kulites each and station 4 had 16 Kulites. At each station Kulites were equally divided among all four faces of the Cable Tray. There were 12 Kulites installed on each of the GO2 pressurization line, the $\mathrm{GH} 2$ pressurization line and the LOX feed-line. They were arranged in sets of four Kulites at 3 different stations: 3,5 , and 6 in figure 3(a). The signals from all Kulites were simultaneously sampled at 20,000/sec using a multi-channel, 24 bit analog to digital converter for various statistical analysis. Checks were made to confirm that the data from all channels were time-synchronized.

It is expected that the modest number of sensors used for calculating sectional forces via integration of the pressure distribution has resulted in an increased uncertainty in the buffet load estimation. The level of uncertainty is different between the rectangular Cable Tray and the circular press- and feed- lines. The flow separation points on the Cable Tray were fixed by its corners; therefore, the fewer number of sensors were deemed sufficient to estimate unsteady forces. The circular geometry of the press and feed lines, on the other hand, allows the separation point to oscillate; resulting in a larger uncertainty. Various earlier reports (refs. 8 and 9) indicate that up to 20 sensors are needed for accurate measurement of the sectional dynamic forces on a circular crosssection. This obviously was impossible to implement in the present test. The $0.25 \mathrm{in}$. diameter press lines only allowed for positioning 4 Kulites at each measurement station. Besides, problems acquiring a large number of Kulites and the time and resource issues limited the total number of sensors. In order to estimate measurement uncertainties, 16 Kulite sensors were placed at one of the instrumented sections of the Cable Tray and 8 on a press-line (both in the LO2 test articles). By progressively using fewer sensors to calculate the root-meansquare (rms) of the unsteady sectional force it was realized 
that fewer sensors resulted in levels higher than actual. Typically, use of 8 sensors on the Cable Tray led to $\sim 20$ percent increase in the calculated rms of the normal and lateral forces compared to those measured from 16 sensors. For the presslines, use of 4 sensors instead of 8 increased levels typically by 50 percent.

\section{Brief Description of LO2 Test Articles and Dynamic Pressure Instrumentation}

The 50 percent scaled LO2 model had two major test articles: a Cable Tray and a Gaseous Oxygen (GO2) pressurization line. They were tested at cross flow angles of $\pm 30^{\circ}$, $+60^{\circ}$, and $+90^{\circ}$. Figure 4 shows the test articles at $90^{\circ}$ cross flow configuration. A total of 42 static pressure taps and 72 high-frequency absolute pressure transducers (Kulite) were installed on the LO2 test article. Of these totals, 48 Kulites were installed on the Cable Tray in groups of 8 at four axial stations and a group of 16 in one axial station. There were 16 Kulites installed on the GO2 pressurization line in two groups of 4 Kulites and one group of 16. Two additional Kulites were installed on each of the Ice/Frost Ramps.

\section{8- by 6-Foot Supersonic Wind Tunnel}

The 8-ft high by 6 - $\mathrm{ft}$ wide perforated wall transonic tunnel (fig. 5) is one side of a closed loop tunnel; the other leg forms a 9- by 15-Foot Low-Speed Wind Tunnel. For the present test the transonic side is used in the aerodynamic (closed loop) mode where air is drawn through the dryer and re-circulated inside the tunnel loop. A cooler in the return leg removes the heat of compression, which allows continuous tunnel operation. The test section length is $23 \mathrm{ft} 6$ in. The tunnel altitude varies from sea level to $36,300 \mathrm{ft}$ as the Mach number varies from 0.36 to 2.0 . The transonic test section is perforated on four sides with 1 -in. diameter holes inclined forward at $60^{\circ}$ and arranged in a herringbone pattern. Various details of the tunnel construction and operation can be found in the users' manual (ref. 7) and the references cited therein.

\section{Incoming Boundary Layer Profiles}

An important aspect of the flow-field is that depending upon the location on the ET, the protuberances are either fully or partially submerged in the thick boundary layer that grows over the long tank surface. Since the forces experienced by the protuberances are expected to be a function of their heights relative to the boundary layer thickness, test data were obtained by varying the gap between the protuberances and the splitter plate, that served as the External Tank surface. Towards that end a boundary layer survey was performed at multiple locations with and without the test articles and at different free stream Mach numbers. Figure 4 shows the locations of the boundary layer rakes and figure 6 shows boundary layer profiles and various other parameters measured ahead of the test articles. The attached turbulent boundary layer is found to thicken at transonic Mach numbers. A comparison of the boundary layer thickness with the LO2 test article shows that the top part of the Cable Tray lay above the undisturbed boundary layer. The Cable Tray and the pressurization lines of the smaller scale LH2 test articles, however, were entirely submerged inside the boundary layer. The large diameter LOX line encountered the largest velocity gradient, with the bottom part lying inside and the top part outside of the incoming boundary layer. The vortical flow shed from the upstream articles and the interaction between various separated regions creates a complex flow-field.

\section{Calculation of Span-Averaged Spectrum of Unsteady Aerodynamic Forces From Measurements of Surface Pressure Fluctuations}

As mentioned earlier the aerodynamic buffet makes only one part of the dynamic loading experienced by the ET protuberances. The other part is the transmitted vibro-acoustic load through the brackets. Each load component has its own spatial and spectral distributions and phase relationships. There exist various in-depth analysis procedures to determine the structural response from the buffet load alone (refs. 10 and 11). However, buffet is not the major source of unsteady loading and needs to be suitably "added" to the other dynamic components. To facilitate calculations it was desirable to determine an average spectrum of the unsteady aerodynamic force fluctuations (buffet) that can be applied uniformly over the entire span between the two attachment points produced by the Ice-Frost Ramps. The calculation process delineated below serves that purpose.

As mentioned earlier, the unsteady sectional forces were measured by groups of dynamic pressure sensors placed on each test article. Since the flow over the test articles are very three-dimensional, the unsteady forces are found to vary significantly over the instrumented span. If the unsteady forces acting at different sections were random and completely uncorrelated then the net force transmitted to the support would be zero. However, an important flow feature was semicoherent vortex shedding which induced partly correlated force fluctuations over the span. Therefore an integral length scale was defined via correlating unsteady forces measured from the adjacent instrumented sections. The sectional forces adjusted by the integral length scale provided a measure of the span-averaged unsteady force. An attempt to apply these ideas to the spectrum of the force fluctuations brought up another issue. The different frequency bands have been found to have different correlation lengths based upon the flow physics involved. Therefore, a frequency-dependent correlation length was introduced and a correlation-adjusted span-averaged spectrum was calculated. There are multiple steps involved in the calculation procedure. Broadly they can be separated into three groups. 
(1) Calculation of the sectional unsteady forces via integration of the pressure sensors data. This was performed at multiple span-wise locations, between two adjacent support brackets, where unsteady pressure sensors were grouped.

(2) Estimation of the span integrated unsteady load using correlation between sectional force fluctuations. The correlation coefficients were calculated either via a frequencyindependent or a frequency-dependent method.

(3) Application of scaling factors to obtain flight, full-scale, dimensional, Power Spectral Density (PSD) of the buffet load. The scaling factors involved are physical dimensions, flight versus wind tunnel dynamic pressure $(q)$ and velocity $(v)$.

Figures 7, 8, and 9 illustrate the numerous steps involved in the calculation.

\section{Sectional Lift/Drag Spectra and Phineas Woods/ Warner Dahm Methods}

The coordinate system used in the following analysis (fig. 7a) uses $x$ as the span-wise direction between support brackets, $z$ as the vertical distance from the splitter plate, and $y$ as the lateral distance normal to the length of the protuberances. The time-dependent pressure fluctuation from each of the pressure sensors (fig. 7) is identified by $p_{i j}(t)$, where $j$ is the station number and $i$ represents a particular sensor in the cluster. At first a suitable length-weighted summation was performed from sensors at $j^{\text {th }}$ cluster to calculate time histories of the sectional normal $N^{j}$ and lateral $L^{j}$ forces:

$$
N^{j}(t)=\sum_{i} p_{i j}(t) \Delta y_{i}, \quad L^{j}(t)=\sum_{i} p_{i j}(t) \Delta z_{i}
$$

Where $\Delta y_{i}$ is the lateral distance between the pressure ports on the cable tray and $\Delta y_{i}$ is the vertical distance (fig. 1(b)). For the press- and feed-lines, which have circular geometry, projected lengths along the normal and the lateral direction were used for the summation process. Since the issue of scaling wind-tunnel data to flight conditions is best dealt with via use of the non-dimensional parameters, all measured data are first normalized to lift and drag coefficients. The time average of the instantaneous forces when normalized by the tunnel free stream dynamic pressure $q_{t}$ and a reference length $l_{\text {ref }}$ provides time averaged sectional lift and drag coefficients at station $j$ :

$$
\bar{C}_{l-a v}^{j}=\frac{\overline{N^{j}}}{q_{t} l_{r e f}}, \quad \bar{C}_{d-a v}^{j}=\frac{\overline{L^{j}}}{q_{t} l_{r e f}} .
$$

The over-bar indicates a time-average. The reference length is the diameter for the feed and press-lines. For the Cable Tray the height was used for the drag coefficient and width for the lift coefficient. The subscript $t$ represents wind tunnel conditions. Similar normalization of the root-mean-square of fluctuating parts (average subtracted) of the forces was used to determine the rms of lift and drag forces:

$$
\widetilde{C}_{l-r m s}^{j}=\frac{\widetilde{N}_{r m s}^{j}}{q_{t} l_{r e f}}, \quad \widetilde{C}_{d-r m s}^{j}=\frac{\widetilde{L}_{r m s}^{j}}{q_{t} l_{r e f}} .
$$

The tilde $(\sim)$ indicates the fluctuating part and the subscript $r m s$ stands for root-mean-square. Figure 8 shows typical average and rms sectional lift and drag variation measured for the LH2 Cable Tray at $30^{\circ}$ cross flow angles. The labels on the $\mathrm{y}$-axis in the figures use slightly different symbols: $\mathrm{Cl}-\mathrm{av}$ and $C d-a v$ to represent the time-average lift and drag coefficients. Similarly Cl-rms and Cd-rms are used to represent the rms fluctuations. Recall that there were five instrumented sections on the Cable Tray at $\mathrm{x} / \mathrm{span}=0.12,0.27,0.41,0.56$, and 0.7 , where span $=$ distance between the centerlines of the adjacent IFRs. Figure 8 shows the span-wise distribution of the mean and fluctuating lift/drag forces for a Mach number range of 0.6 to 1.6. Note the advantage of using lift and drag coefficients (as opposed to the dimensional form) is the near superposition of all Mach number data.

The Power Spectral Density (PSD, expressed by $\Phi$ ) of unsteady force fluctuations were calculated via digital Fourier transform of force time-histories. For the normal force component it is expressed as:

$$
\Phi_{N}^{j}\left(f_{t}\right)=\frac{G_{N^{j}} G_{N^{j}}^{*}}{\Delta f_{t}},
$$

Where $G_{N^{j}}$ is the Fourier transform of the time-varying sectional force $N^{j}$ at the $j$ th station. The superscript $*$ represents the complex conjugate, and $\Delta f_{t}$ is the width of the frequency band in the digital transform. The long time history acquired during the test was segmented into smaller strings of $n=2048$ points; each string was Fourier transformed and the final PSD estimate is an average over all segments. The Nyquist criterion provides the width of the frequency band:

$$
\Delta f_{t}=\frac{2}{\Delta t n}, \quad \frac{1}{\Delta t}: \text { sampling rate }
$$

For the spectral data, frequency is normalized to Strouhal number $S_{t}$ :

$$
S_{t}=\frac{f_{t} l_{r e f}}{U_{t}}
$$

The PSD of the fluctuating sectional lift-coefficient is calculated from that of the dimensional lift force based on the requirement that an integration of PSD should be equal to the mean square of the time series. The PSD for the fluctuating lift-coefficient is the following: 


$$
\Phi_{c l}^{j}\left(S_{t}\right)=\frac{U_{t}}{q_{t}^{2} l_{r e f}^{3}} \Phi_{N}^{j}\left(f_{t}\right)
$$

Figure 9(a) shows spectra of the sectional lift-coefficient for the Cable tray. This report presents all model scale force data in terms of average, rms and PSD of lift/drag coefficients.

The sectional lift/drag forces were found to vary significantly over the span-wise distance $x$ between the Ice/Frost ramps. The goal of the program was to provide an average spectrum of the sectional forces. However, that can not be achieved by a simple averaging of the spectra as the unsteady forces at various measurement stations may be only partly correlated. To include the effect of spatial correlation a method proposed by Phineas Wood (ref. 3) and Warner Dahm (ref. 2) was expanded upon. The basic premise of the method is that an average sectional force (say lift) $\overline{\widetilde{C}}_{l-r m s}$ over a subsegment $x_{s}$ (figure $7(\mathrm{a})$ ) can be expressed via an integral correlation length $l_{c}^{x_{s}}$ calculated from the correlation coefficient of forces between adjacent locations:

$$
\overline{\widetilde{C}}_{l-r m s}=\widetilde{C}_{l-r m s}^{j} \frac{l_{c}^{x_{S}}}{l_{\text {span }}}=\widetilde{C}_{l-r m s}^{j} \frac{1}{l_{\text {span }}} \int_{0}^{x_{S}} r(x, \tau=0) d x
$$

Here $r$ is the correlation coefficient between fluctuating forces at two adjacent stations $j$ and $j+1$, and $\tau$ is the correlation time or the delay time. The correlation length $l_{c}$ is normalized by the segment length of the protuberances $\left(l_{\text {span }}\right)$ between the adjacent supporting brackets (IFR). Once again, the following expression is for the normal (lift) component of the force:

$$
r(x, \tau)=\frac{1}{\widetilde{N}_{r m s}^{j} \tilde{N}_{r m s}^{j+1}} \int_{0}^{T} \tilde{N}^{j}(t) \tilde{N}^{j+1}(x, t+\tau) d t
$$

$T$ is the time duration of data used to calculate the correlation coefficients. Figure 9(b) shows correlation in normal force fluctuations between pairs of adjacent instrumented stations. There were five instrumented stations; therefore, correlation was calculated between four pairs of adjacent stations. Since force fluctuations were measured in a limited few stations, some modeling of the spatial variation of the correlation coefficient $r$ needs to be introduced. The commonly used exponential decay model (refs. 9 and 12) is a reasonable choice:

$$
r=\exp \left(-x / l_{c}\right), \text { such that } \int_{0}^{\infty} r d x=l_{c}
$$

If the correlation length $l_{c}>l_{\text {span }}$ : the distance between support brackets, then $l_{c} l_{\text {span }}=1$, i.e., the forces over the entire span are correlated. The integral in equation (8) becomes equal to $l_{c}$. Additionally, via the use of $x / l_{\text {span }}$ in equation (10) the first part of equation (8) rewritten as:

$$
\overline{\widetilde{C}}_{l-r m s}=\widetilde{C}_{l-r m s}^{j} \frac{l_{c}}{l_{\text {span }}}
$$

also signifies that if the sectional forces are uncorrelated $l_{c}<<1$, then effectively the average unsteady force experienced by the supporting brackets will also be minimal. The above relation is easy to extend to determine the PSD of the lift fluctuations. The average PSD over a part of the test article can be estimated from the sectional PSD $\Phi_{c l}^{j}$ as the following:

$$
\bar{\Phi}_{c l}\left(S_{t}\right)=\Phi_{c l}^{j} \frac{l_{c}}{l_{\text {span }}}
$$

Equations 11 and 12 are primary results of Phineas Wood's derivation. Since for the most part $l_{c}<l_{\text {span }}$, the span averaged spectrum is called the lift spectrum including correlation knockdown. Physically, it says that only the correlated part of the force over a segment contributes to the average, while the uncorrelated part has little contribution. Some modeling schemes were introduced to apply equation (12) to the test articles with $k$ number of span-wise measurement stations $(j=1,2, . . k)$ and significant flow non-uniformities. Only two adjacent stations with spacing $\Delta x$ were used to estimate a correlation length:

$$
l_{c}^{j}=-\frac{\Delta x}{\ln (r)}, \quad j=1,2, \ldots(k-1)
$$

Once again this is based upon the assumption of exponential decay of the correlation coefficient $r$ with separation distance $\Delta x$. An average of all such correlation length was used for the final estimation.

$$
\frac{\bar{l}_{c}}{l_{\text {span }}}=\frac{1}{k-1} \sum_{j=1}^{k-1} \frac{l_{c}^{j}}{l_{\text {span }}}
$$

The black, straight line in figure 9(e) is the average correlation length calculated via averaging values obtained from the adjacent measurement stations.

\section{Narrowband (Frequency Dependent) Correlation Length}

Note that correlation length used in equations (8) through (14) is a frequency-independent fixed number; therefore, it is 
also called frequency independent correlation length. The lift/drag spectrum provides distribution of energy in narrow frequency bands. The narrow-band description provides additional information on coherent vortex shedding, shock unsteadiness etc. Therefore, formulation of a narrowband, frequency-dependent correlation is desirable. Additionally, Dahm clearly warns against using the frequency-independent correlation coefficient for situations where periodic vortex shedding may exist. In various turbulence shear flow analyses, frequency-dependent correlation length is calculated via coherence spectrum. Instead of the correlation coefficient $r$, a coherence spectrum is calculated from Fourier transforms of force time-histories at stations $j$ and $j+1$ :

$$
\Gamma_{N}^{j}\left(S_{t}\right)=\sqrt{\frac{\left|G_{N^{j}} G_{N^{j+1}}^{*}\right|}{\left|G_{N^{j}}\right|\left|G_{N^{j+1}}^{*}\right|}} .
$$

Note that the square root ensures simple magnitude, rather than magnitude-squared, coherence spectrum. Figure 9(c) shows the magnitude squared coherence calculated from adjacent measurement stations. The numerator of the above equation contains the magnitude of the cross-spectrum. The existing buffet literatures (refs. 10 and 11) also uses the real part (co-spectrum) and the imaginary part (quad-spectrum) of the cross-spectrum separately. It is easy to calculate these two components from knowledge of the cross-spectral phase shown in figure $9(\mathrm{~d})$.

In some ways, the coherence spectrum represents a spectral distribution of the correlation coefficient. If each of the narrow-frequency bands is assumed to decay exponentially over spatial separation $\Delta x$ then equation (13) can be expanded to define the desired spectrum of frequency-dependent correlation length:

$$
l_{c}^{j}\left(S_{t}\right)=-\frac{\Delta x}{\ln \left(\Gamma_{N}^{j}\right)}, \quad j=1,2, \ldots(k-1)
$$

An average of all such data from the adjacent stations provides an average of the frequency-dependent correlation length:

$$
\frac{\bar{l}_{c}\left(S_{t}\right)}{l_{\text {span }}}=\frac{1}{k-1} \sum_{j=1}^{k-1} \frac{l_{c}^{j}\left(S_{t}\right)}{l_{\text {span }}}
$$

The correlation lengths calculated from using (14) and (17) are shown in figure 9(e) for the example case under study.

\section{Span-Averaged, Sectional Load Spectrum}

Following the methodology suggested by equation (12), multiplying average spectrum of sectional forces by averaged correlation length provides an average spectrum of the unsteady buffet load. PSD of sectional forces are known at multiple stations, an average of all these measurements was used before performing the multiplication. Additionally, there are two available choices of correlation lengths: either a frequency independent fixed value, or a narrowband, frequency-dependent spectrum. The final PSD can be computed in two different ways.

$$
\begin{gathered}
\bar{\Phi}_{c l-f i x e d}\left(S_{t}\right)=\left(\frac{1}{k} \sum_{j=1}^{k} \Phi_{c l}^{j}\right) \frac{\bar{l}_{c}}{l_{\text {span }}} \\
\bar{\Phi}_{c l}\left(S_{t}\right)=\left(\frac{1}{k} \sum_{j=1}^{k} \Phi_{c l}^{j}\right) \frac{\bar{l}_{c}\left(S_{t}\right)}{l_{\text {span }}}
\end{gathered}
$$

The lift spectrum of equation (18a) uses the fixed, frequency independent correlation length while (18b) uses the narrowband correlations.

At this point it is worthwhile to discuss the conservativeness of the buffet load estimation using the above two choices of correlation lengths. The issue emanates from a fundamental question of what to do with the phase relationship between the sectional-force fluctuations measured from the adjacent stations (figure 9(d)). Equivalently, in the time domain, how to account for the fact that peak correlation does not appear at zero time delay $(\tau)$ but at some non-zero interval (figure $3(b)$ ). The Woods-Dahm formulation in equation (8) neglects this time delay. The frequency independent correlation length is calculated based on very small correlations measured at $\tau=0$. This obviously provides a shorter correlation length; thereby a lower spectrum level is calculated via the use of equation (18a). Physically the large scale vortex shedding, leading to the larger correlation coefficient, occurs with a time/phase delay along the span of the test articles. The corresponding sectional force generated from the shedding are not in phase; and therefore, may lead to cancellation when added together. However, from the point of view of exciting structural modes, two periodic forces with $180^{\circ}$ phase difference, but at the correct resonance frequency can excite a bending mode. The sum of these two forces could be very small, yet their combined effect can cause large deflections. Since the goal of the present test was to provide an estimate of the forcing function ignoring structural response, a more conservative estimate of force is desirable.

The frequency dependent correlation, defined via coherence spectrum, on the other hand, is based on the peak value of correlation and provides a larger, conservative estimate of the unsteady sectional force. Fundamentally, cross-spectrum and cross-correlation are related via Fourier transform. The coherence function of equation (15) is based on the magnitude of cross-correlation which corresponds to the peak value of correlation (the time delay information appears as crossspectral phase, and are not used in the present formulation). Typically, in broad-band fluctuations, correlations at $\tau=0$ is 
representative of the high frequency component $(f \rightarrow 1 / \tau)$. A closer look into figure 3(e) indeed demonstrates that the frequency independent correlation closely follows the high frequency tail of the narrowband correlation spectrum. The longer correlation length associated with vortex shedding (around $S_{t}=0.1$ ) are mostly missed.

The downside of using the frequency-dependent correlation length is the longer computational time required to process the large number of data sets. Therefore, to produce the Critical Design Review (CDR) environment a simplistic process was used by the program team. In addition to the frequency independent correlation length of equation (14), a band-passed correlation length was calculated over the frequency range where vortex shedding is expected.

\section{Scaling to Flight Condition}

Equation 18(b) provides an average spectrum of nondimensionalized fluctuations. Once this non-dimensional spectrum is determined, calculation of the absolute force spectrum in dimensional form $(\mathrm{lb} / \mathrm{in} \text {. })^{2} / \mathrm{Hz}$ for the full scale vehicle takes a straight forward path. The fundamental premise of the scaling law is that the Strouhal frequencies, the fluctuating lift and drag coefficients, and the dimensionless correlation lengths will remain the same between the model and the full-scale. The flight velocity $V_{f}$ and the dynamic pressure $q_{f}$ were provided from Computational Fluid Dynamic analysis of the full-stack vehicle at different free-stream Mach numbers. The full-scale segment length $l_{\text {span full }}$ and crosssectional dimension $l_{\text {ref full }}$ of the individual protuberances were known from the scale factor and model scale dimensions. Using the inverse of equation (7) the dimensional, full scale, sectional normal load PSD is obtained as:

$$
\begin{aligned}
& \bar{\Phi}_{N-\text { full }}\left(f_{f}\right)=\frac{q_{f}^{2} l_{r e f_{-} \text {full }}^{3}}{V_{f}} \bar{\Phi}_{c l}\left(S_{t}\right), \\
& f_{f}=\frac{S_{t} V_{f}}{l_{\text {ref_full }}}
\end{aligned}
$$

Figure 9(f) shows a full-scale, dimensional sectional lift spectrum that is averaged over the span between IFRs and scaled up to the flight conditions. This concludes the first part of the manuscript.

\section{PAL Ramp On Versus Off}

The bulk of the test data was collected for no-PAL ramp configurations. Only a limited amount of test data was collected with the PAL ramps in place. One case of the LO2 test articles at $90^{\circ}$ cross flow and one case of the $\mathrm{LH} 2$ articles with $30^{\circ}$ cross-flow were studied with the PAL ramp placed ahead of the test articles. The corresponding data points without the
PAL ramp were also acquired. Figure 10 shows schematics of the LO2 PAL ramp as mounted on the splitter plate. Corresponding schematic for no-PAL ramp configuration was presented earlier in figure 4. The LO2 ramp was as tall as the top edge of the Cable Tray. This provided an effective protection from cross-flow induced force fluctuations. Figure 11 provides a comparison of the steady and unsteady lift and drag forces encountered by various LO2 test articles. No attempt was made to calculate span-averaged spectra, as outlined in the previous section, for this comparative study. The rms values and model-scale spectra were deemed sufficient to establish the change in the unsteady buffet loading. An examination of data shows that both steady and unsteady forces are diminished by a factor of 3 to 10 when the PAL ramp is in place. A closer look into $\mathrm{Cl}$-rms and $\mathrm{Cd}$-rms plots shows that at the two Mach number conditions of $\mathrm{M}=0.6$ and 1.08, PAL ramp data have relatively higher fluctuations than all other Mach conditions. Upon examination of the corresponding spectra it was found that there were strong tones at $5000 \mathrm{~Hz}$ to $7000 \mathrm{~Hz}$ present at certain Mach number conditions. The source of the tones was conjectured to be the narrow gap between the PAL ramp and the Cable Tray (fig. 10(b)) which perhaps helped setup a cavity resonance phenomenon. Usually tonal behaviors are absent in rocket flights as the fast changing flight velocity does not allow sufficient time for setting up the necessary feed-back loop. Nevertheless, in spite of the presence of the tone in the component wind tunnel test, the LO2 PAL ramp was found to be effective in reducing steady and unsteady aerodynamic forces.

Figure 12 shows a schematic of the LH2 PAL ramp; the corresponding no PAL configuration can be found in figure 3 . The LH2 PAL ramp was again of the same height as that of the Cable Tray. However, it was far smaller than the large diameter LOX feed-line. Additionally, the feedline and the pressurization lines were significantly separated from the Cable Tray (fig. 3(c)). All of these made it unlikely that the LH2 PAL ramp would have any significant impact on all but the Cable Tray. Figure 13 presents a comparison of the steady and unsteady lift and drag forces on all four protuberances with and without the ramp. As expected the influence of the PAL ramp was mixed. Since the Cable Tray and the GO2 press-line lay close to the ramp, the reduction is most visible for these two elements. The $\mathrm{GH} 2$ pressline and the LOX feedline lay further away; aerodynamic forces on these elements are hardly influenced by the presence of the LH2 PAL ramp. An examination of force fluctuations spectra (not presented) also indicated that the LH2 PAL ramp was ineffective in stopping quasi-periodic vortex shedding.

\section{Concluding Comments}

The protuberances on the External Tank are long cylindrical and rectangular rods that are periodically supported on the tank surface. They are either fully or partially submerged into the thick boundary layer formed over the tank. Computational 
simulation of the flow-field showed complex threedimensional patterns between the adjacent supports. The experimentally measured spectra of unsteady force fluctuations showed that even in between two adjacent supports there are multiple flow features, such as coherent vortex shedding, low frequency unsteadiness associated with local separated regions, and in general random turbulent fluctuations. The first part of the present paper describes a methodology that is an improvement over the earlier methods of establishing an average spectrum of the buffet load when multiple flow features are manifested in sectional force-spectra. The introduction of frequency-dependent correlation lengths is expected to correctly adjust different frequency bands for a wide range of correlation lengths.

The second part of the paper describes the difference of the unsteady buffet forces with and without the PAL ramps. The LO2 test articles are far more affected by the removal of the ramp. However, the buffet forces are only a part of the net force encountered by the protuberances; the majority appeared from transmitted vibration through the mounting support. The combined effect of all the forces could only be measured in actual flight. Accelerometers were placed inside the Cable Tray for three different Shuttle Flights. The analysis of data collected from the flight sensors are provided in a companion paper (ref. 13).

\section{References}

1. John W. Edwards, Donald F. Keller, David M. Schuster, David J. Piatak, Russ D. Rausch, Robert E. Bartels, Thomas G. Ivanco, Stanley R. Cole, and Charles V. Spain, "Aeroelastic Response and Protection of Space Shuttle External Tank Cable Trays," AIAA-2005-3627, July 2005.

2. Dahm, K. Werner, "Composite model of a random forcing function for the excitation of long pipes by a cross flow," NASA Marshall Memo no. ED31-79-15, Aug. 1979.
3. Phineas Woods (handwritten notes) "Sample Calculations for ET LO2 Feedline Loads (Generated by Random Vortex Shedding and Aeroacoustic Pressure Fluctuations), Nov. 1979.

4. Boeing Company Space Exploration "Post-test report: component unsteady airloads wind tunnel test of the Space Shuttle External Tank Cable Tray models 207-T (0.5-scale LO2) \& 208-T (0.25-scale LH2) at NASA GRC 8- by 6-ft Supersonic Wind Tunnel (IS-23)," document no. NS06HOU315, Oct. 20, 2006.

5. Karl F. Neuman \& Richard R. Burrows, "Pretest information: Component unsteady airloads wind tunnel test of the Space Shuttle External Tank cable tray models 207-T (0.5 scale LO2) \& 208-T (0.25 scale LH2) at NASA GRC 8by 6-ft Supersonic Wind Tunnel." Document number NS06HOU130A, prepared by: the Boeing Company Space Exploration; revision March 6, 2006.

6. Boeing post-test report.

7. Soeder, R.H. "NASA Lewis 8- by 6-Foot Supersonic Wind Tunnel User Manual," NASA TM-105771, Feb. 1993.

8. Schmidt, L.V. "Measurement of fluctuating air loads on a circular cylinder," AIAA J. of Aircraft, 2(1), 1965, pp. 49-55.

9. Szechenyi, E. "Supercritical Reynolds number simulation for two-dimensional flow over circular cylinders," $J$. Fluid Mech., 70(3), 1975, pp. 529-542.

10. Cockburn, J.A., and Robertson, J.E., "Vibration response of spacecraft shrouds to in-flight fluctuating pressures," $J$. Sound \& Vib., 33(4), pp. 399-425.

11. Himelblau, H., Fuller, C.M., and Scharton, T.D. "Assessment of space vehicle aeroacoustic vibration prediction, design and testing," NASA CR-1596, July 1970.

12. Panda, J., "Two Point Space-Time Correlation of Density Fluctuations Measured in High Velocity Free Jets," AIAA2006-0006, Jan. 2006.

13. Walker, B., Panda, J., and Sutliff, D., "Space Shuttle External Tank Cable Tray Vibration Sensors Flight Measurement Data Analysis," AIAA-2008-0312, Jan. 2008. 


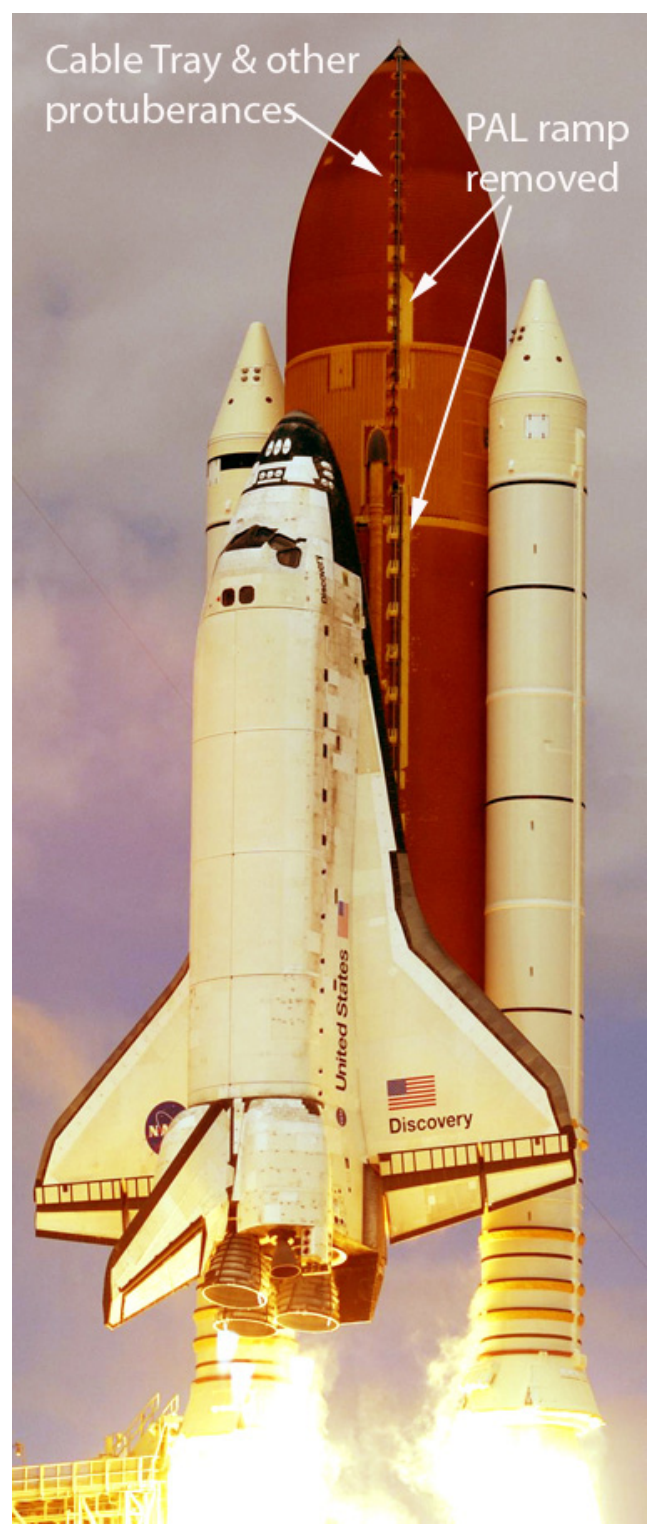

Figure 1.-Location of the PAL ramp and the protuberances on the External Tank.

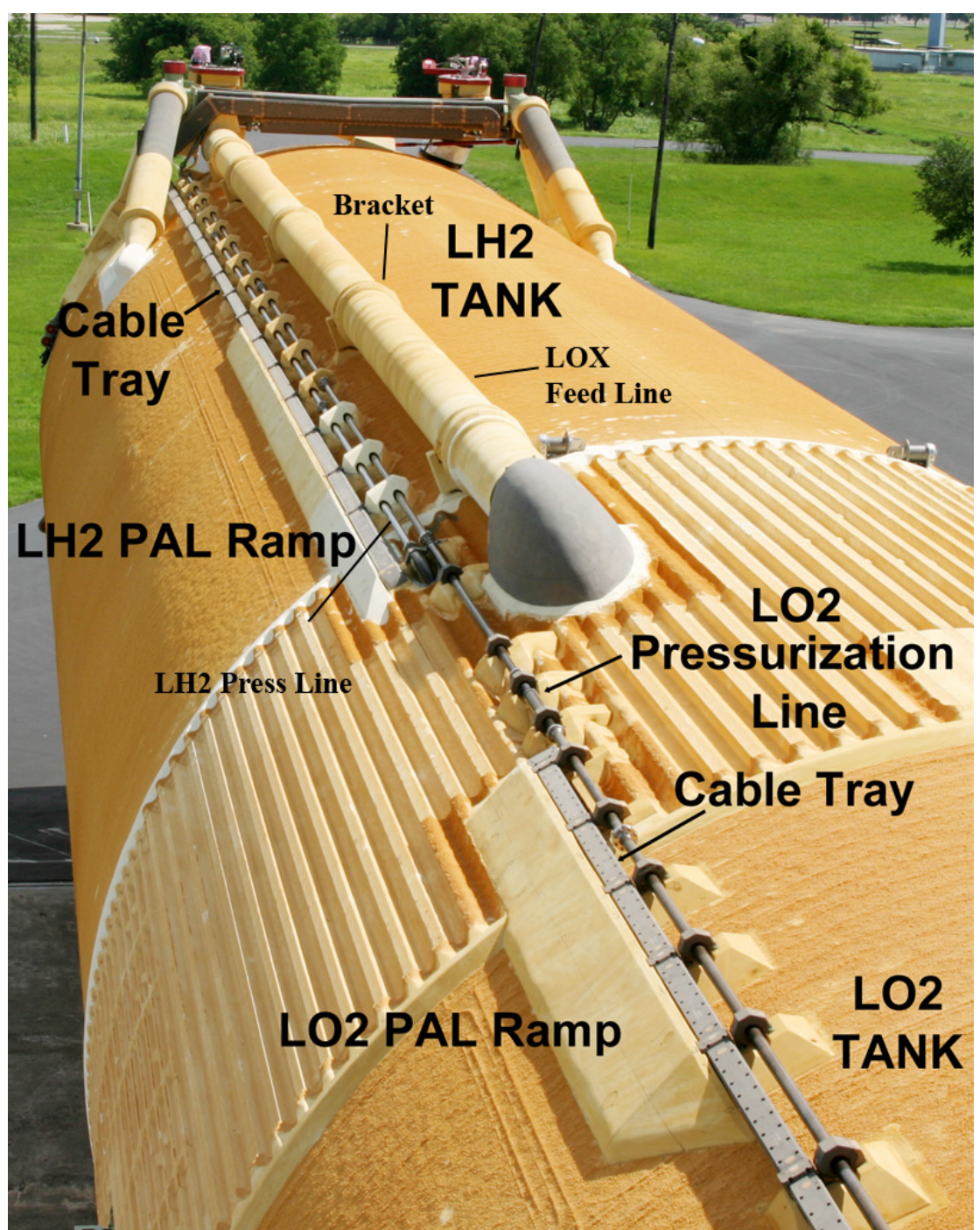

Figure 2.-Close-up view of the External Tank. The section of the LO2 protuberances tested in IS-23 corresponded to those next to the PAL ramp upstream of the serrated inter-tank zone. The section of the $\mathrm{LH} 2$ protuberances corresponds to those in the vicinity of the second bracket on the LOX feedline. 

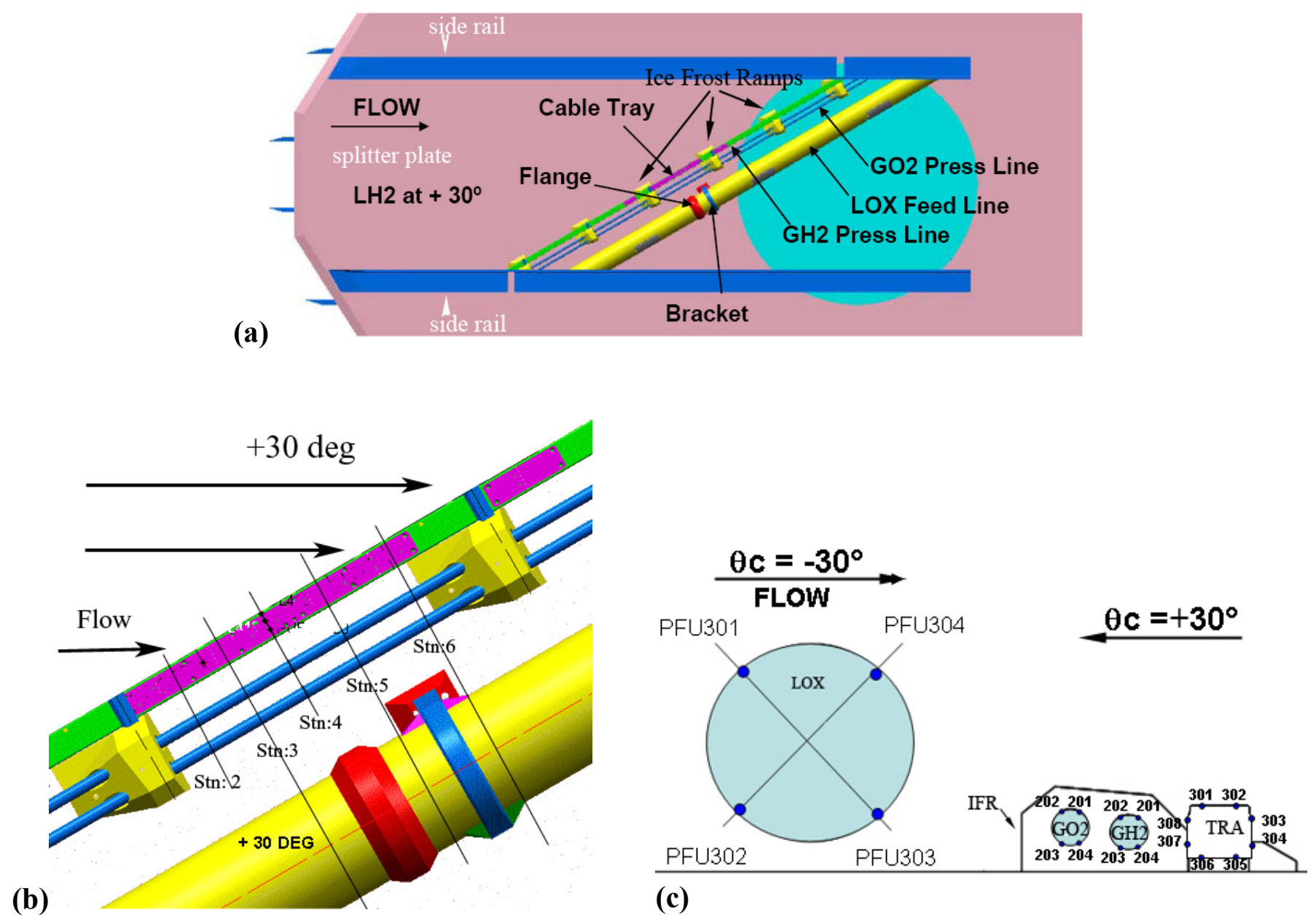

(c)

Figure 3.-(a) LH2 test articles (without PAL ramp) mounted on a splitter plate with side-rails. The configuration simulated $30^{\circ} \mathrm{cross}$ flow angle (CFA), (b) close-up view, and (c) cross-sectional view of the test articles and locations of some dynamic pressure sensors.

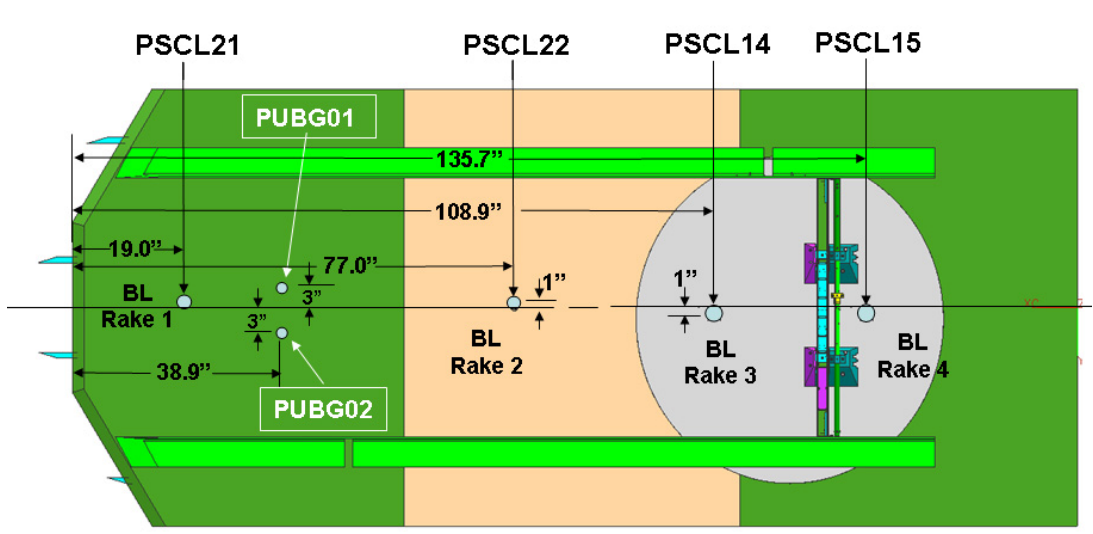

(a)

Figure 4.-LO2 test articles (a) without PAL ramp at $90^{\circ}$ cross flow angle, and (b) cross-sectional view with old IFR. Mounting locations for boundary layer rakes and the associated static pressure ports (marked PSCL21, 22, 14, 15) are shown. The locations of the dynamic pressure sensors (marked as PUBG01, 02) to measure the tunnel background acoustic level are shown in part (a). 


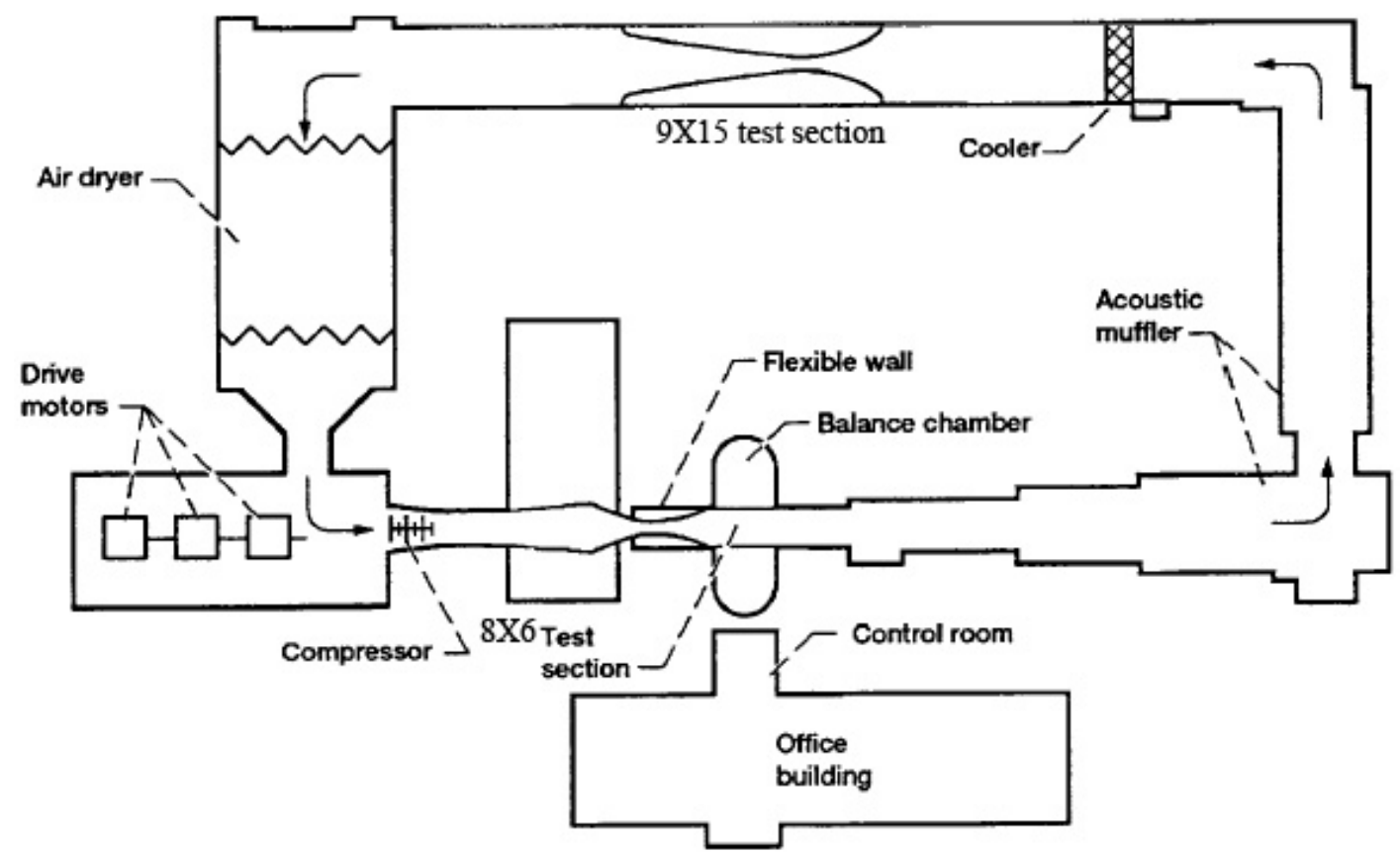

Figure 5.-Schematic of the 8- by 6-Foot transonic wind tunnel.
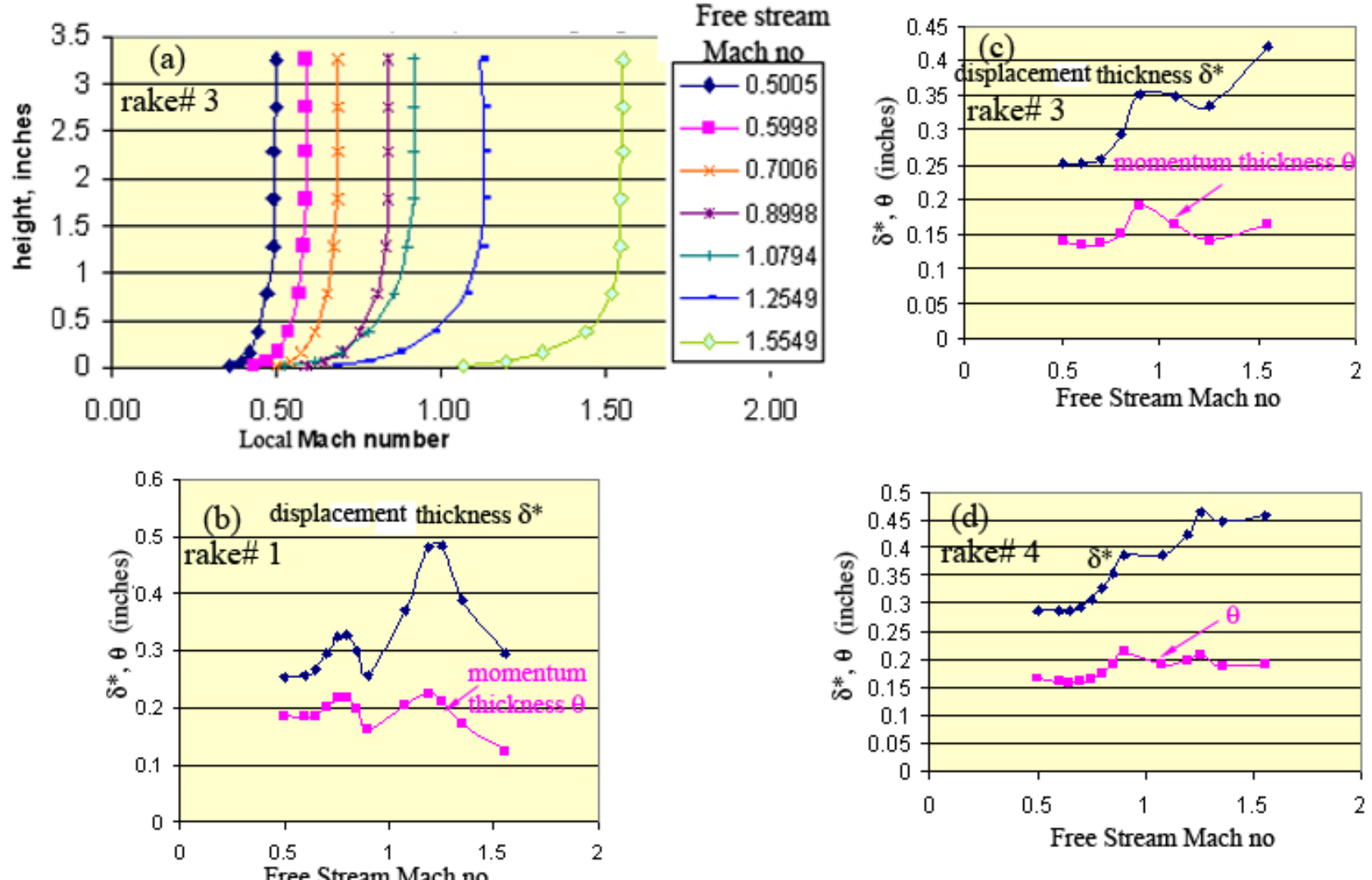

Figure 6.-(a) Boundary layer profiles. (b), (c), and (d) associated displacement and momentum thicknesses at indicated rake locations measured on the splitter plate without the test articles. Rake \#3 was located just upstream of the model and rake \#4 downstream. Rake \# 1 was close to the splitter plate leading edge (see fig. 4(a)). This figure was provided by John D. Saunders and Lance E. Foster of NASA Glenn. 

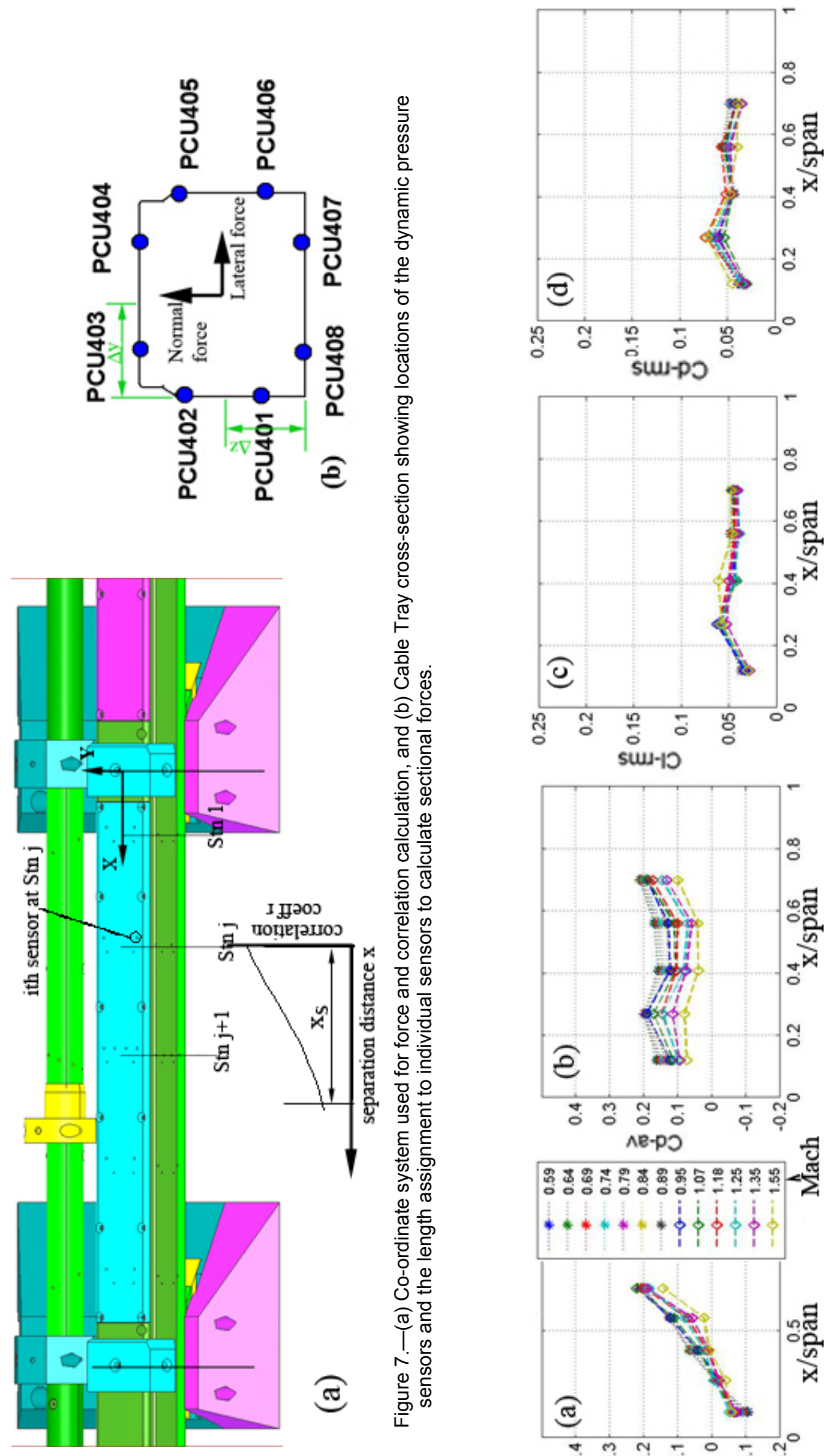

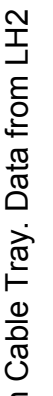
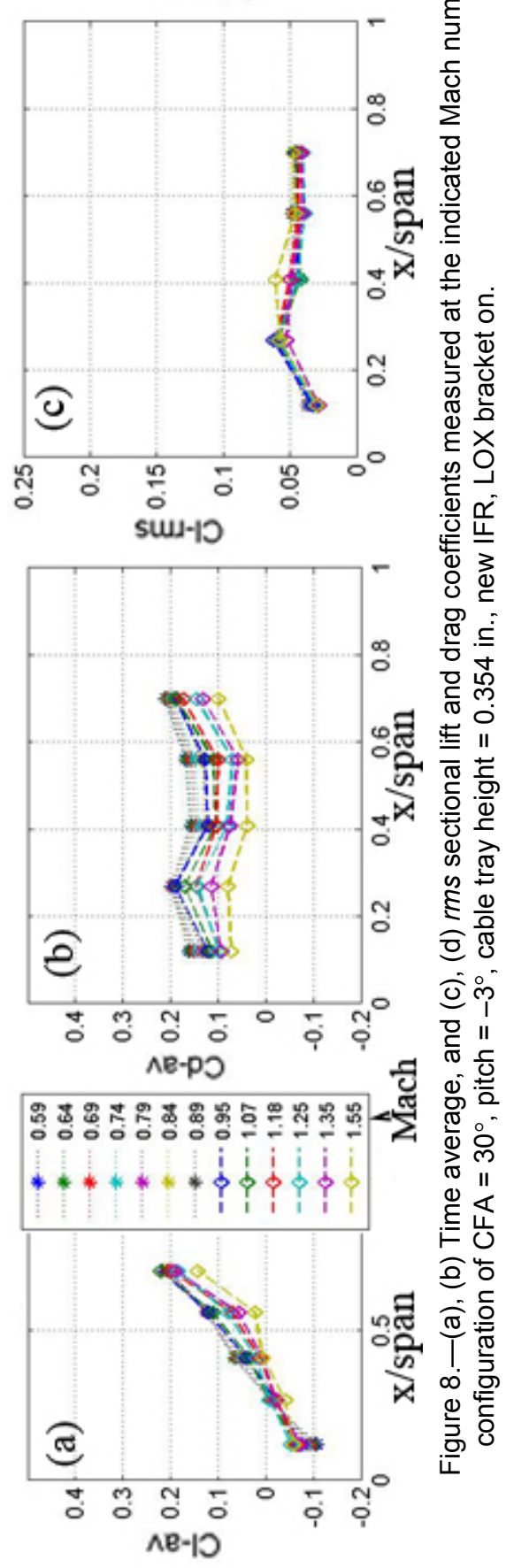

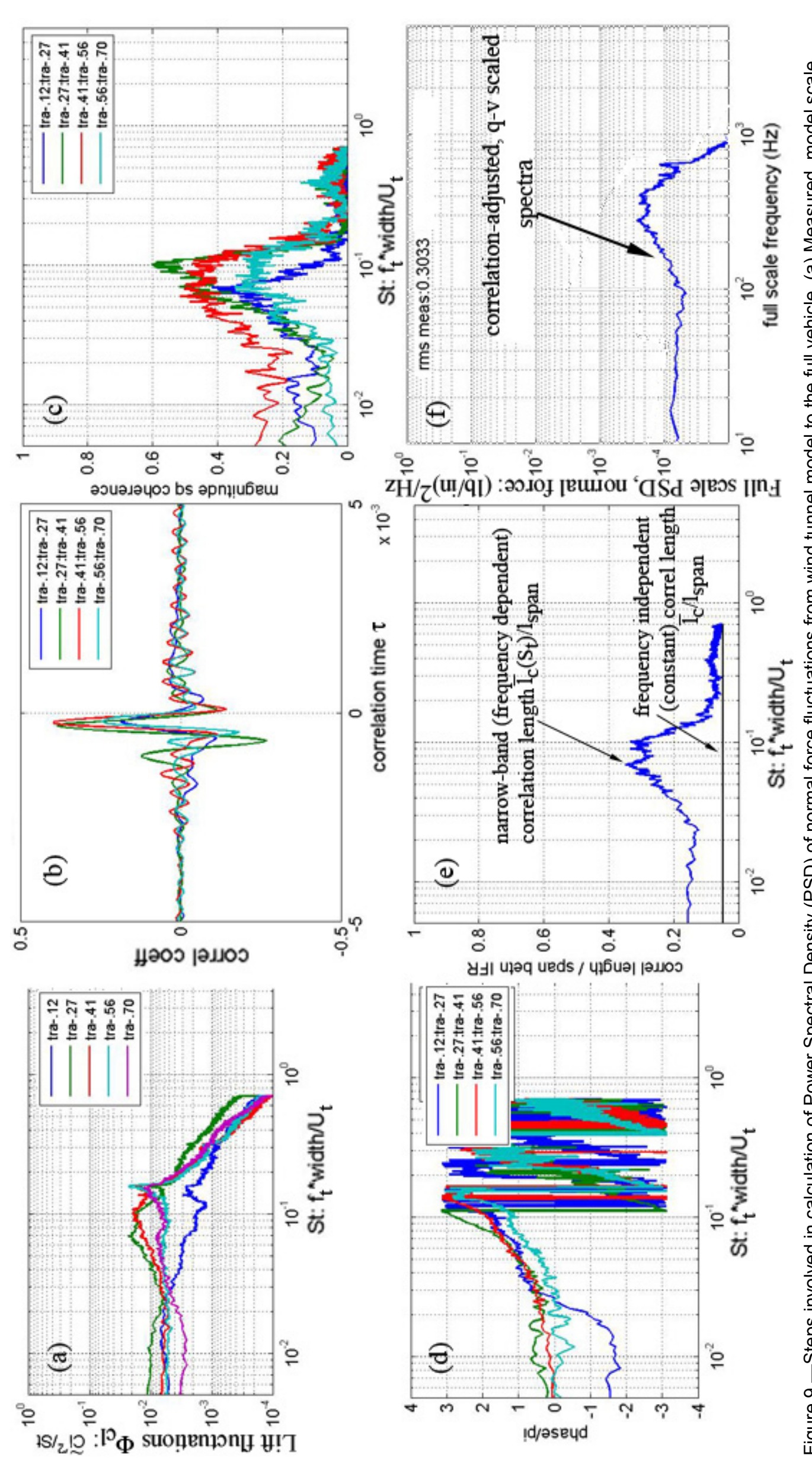

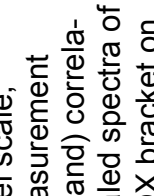

ब。

$\varepsilon$ 年

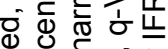

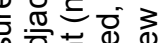

क्ष

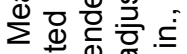

잉

ब

일 ब

菏

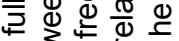

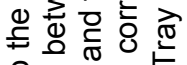

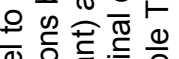

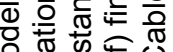

륭잉

ब은 흔 흥

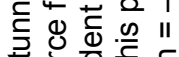

응 응

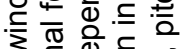

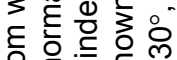

은

क. $\cong$ 造

응 $\frac{\Phi}{2}$

क

过

ग

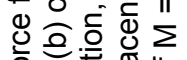

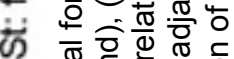

$\bar{\Phi} \Phi \overline{\bar{\sigma}}$

응

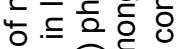

西

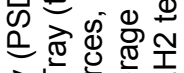

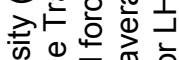

중

吕

중 응

ज्ञ

कि 은

क苋 응

象

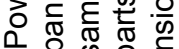

$4 \frac{0}{\times} 0 \geq \frac{1}{2}$

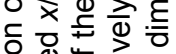

造范

즐 에의

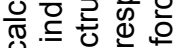

$\subseteq$ 잉 के

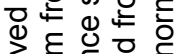

>

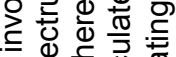

\&

Q क 000

की

ब.

造

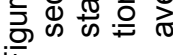



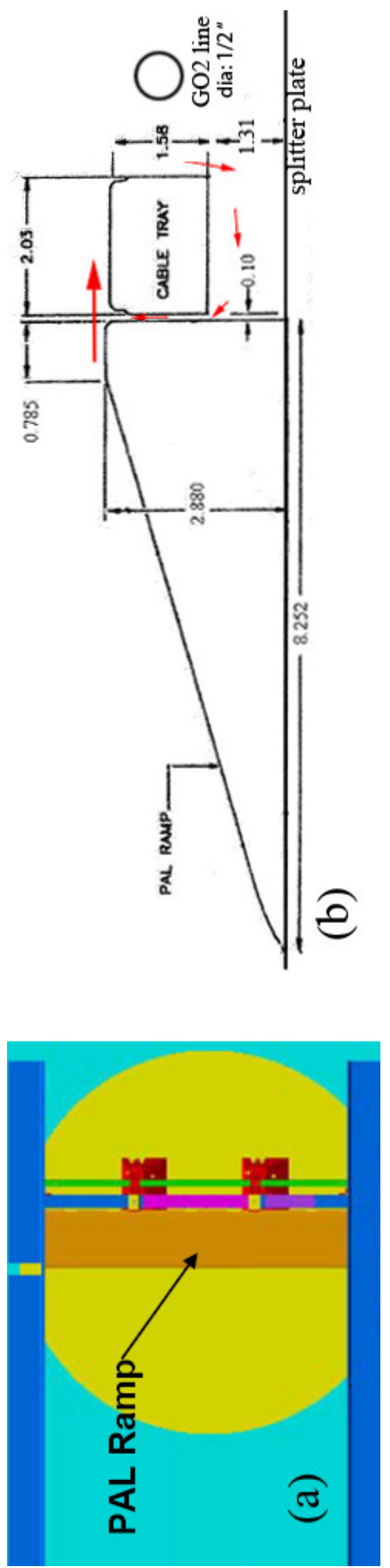

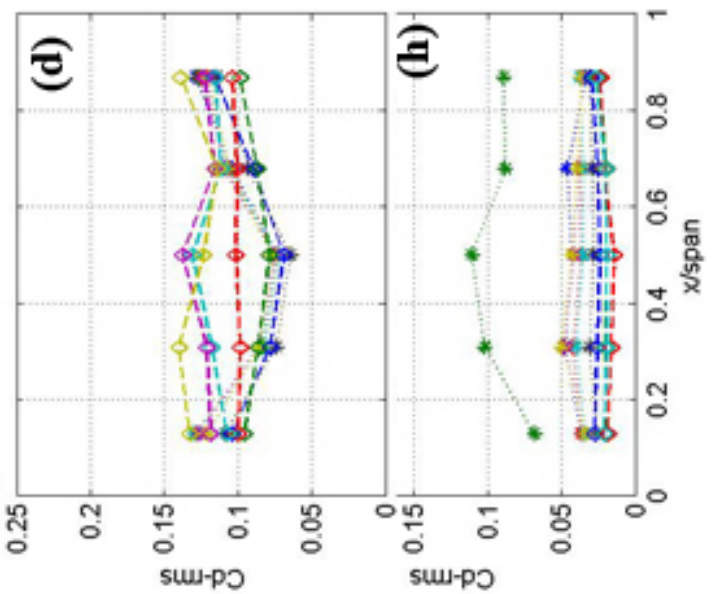

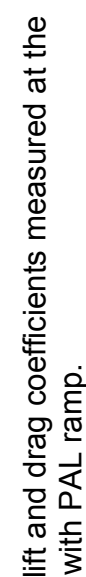

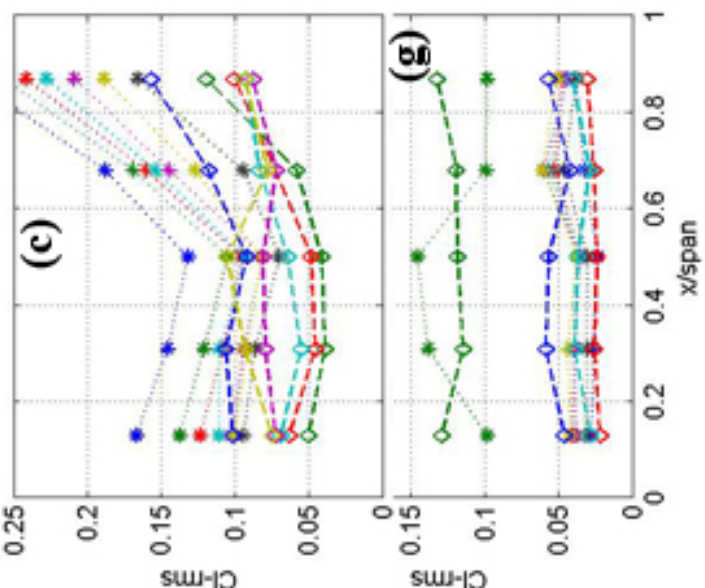

등 응

氖

है

ণั ฮั 지 它 $\frac{1}{2}$ ๙

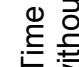
3 은 을 कृ त

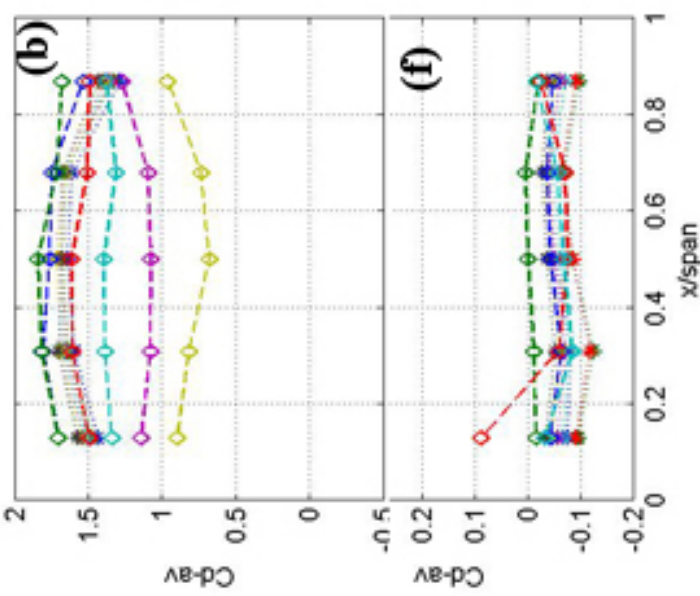
ㅇํㅇ 흘 is कृ है

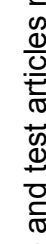
हूำ 压 กิ $\frac{1}{4}$

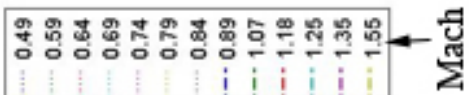
********)\}\{\$\}?

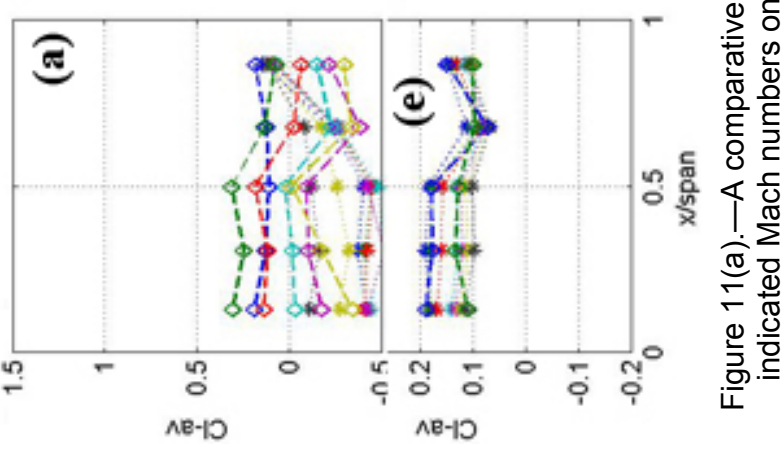



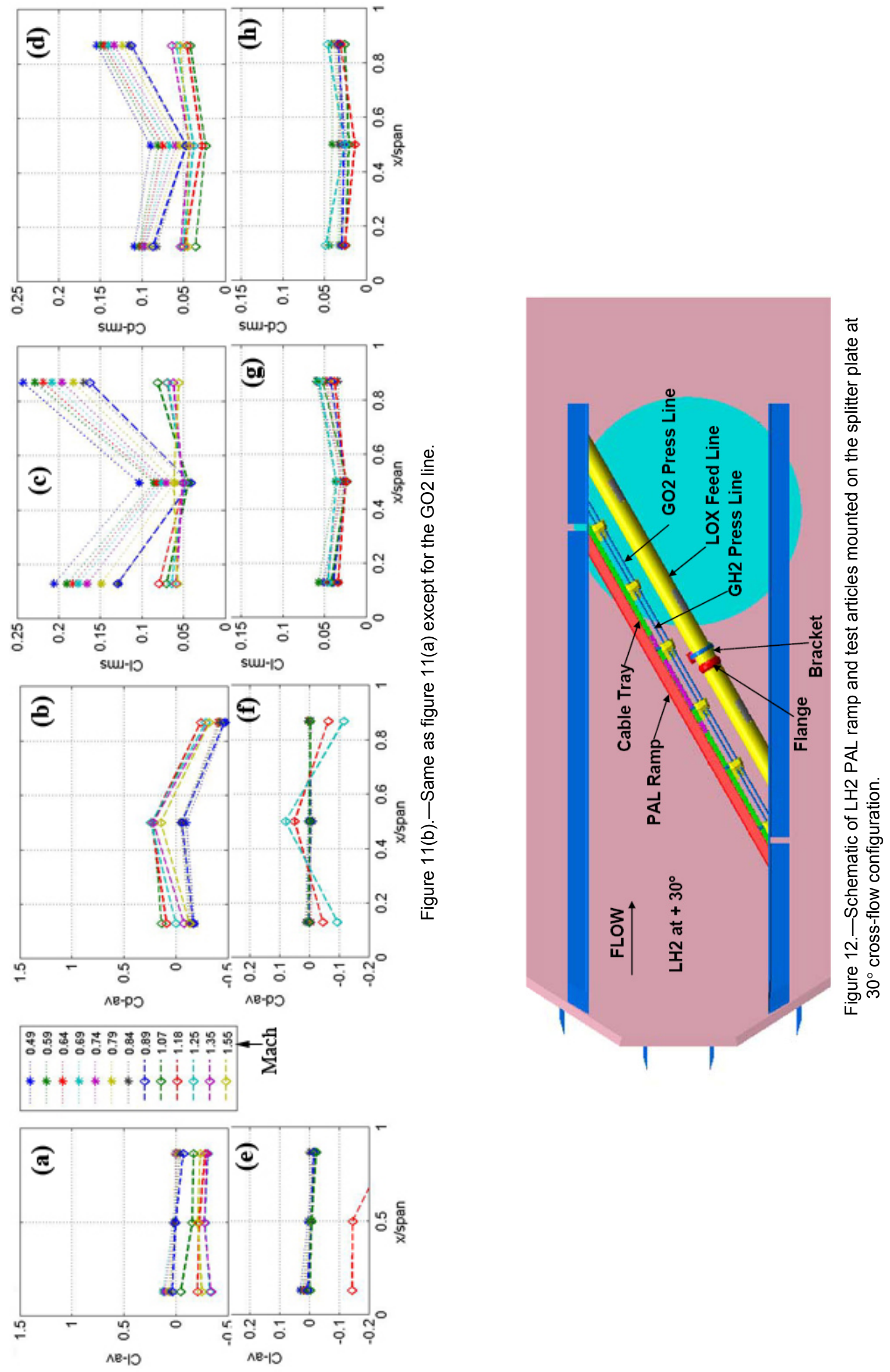


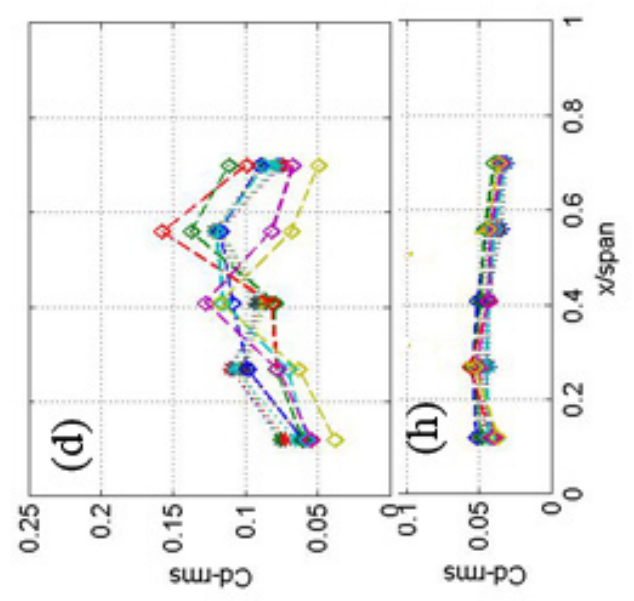

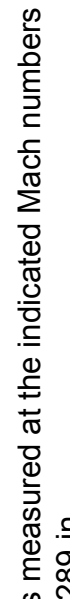
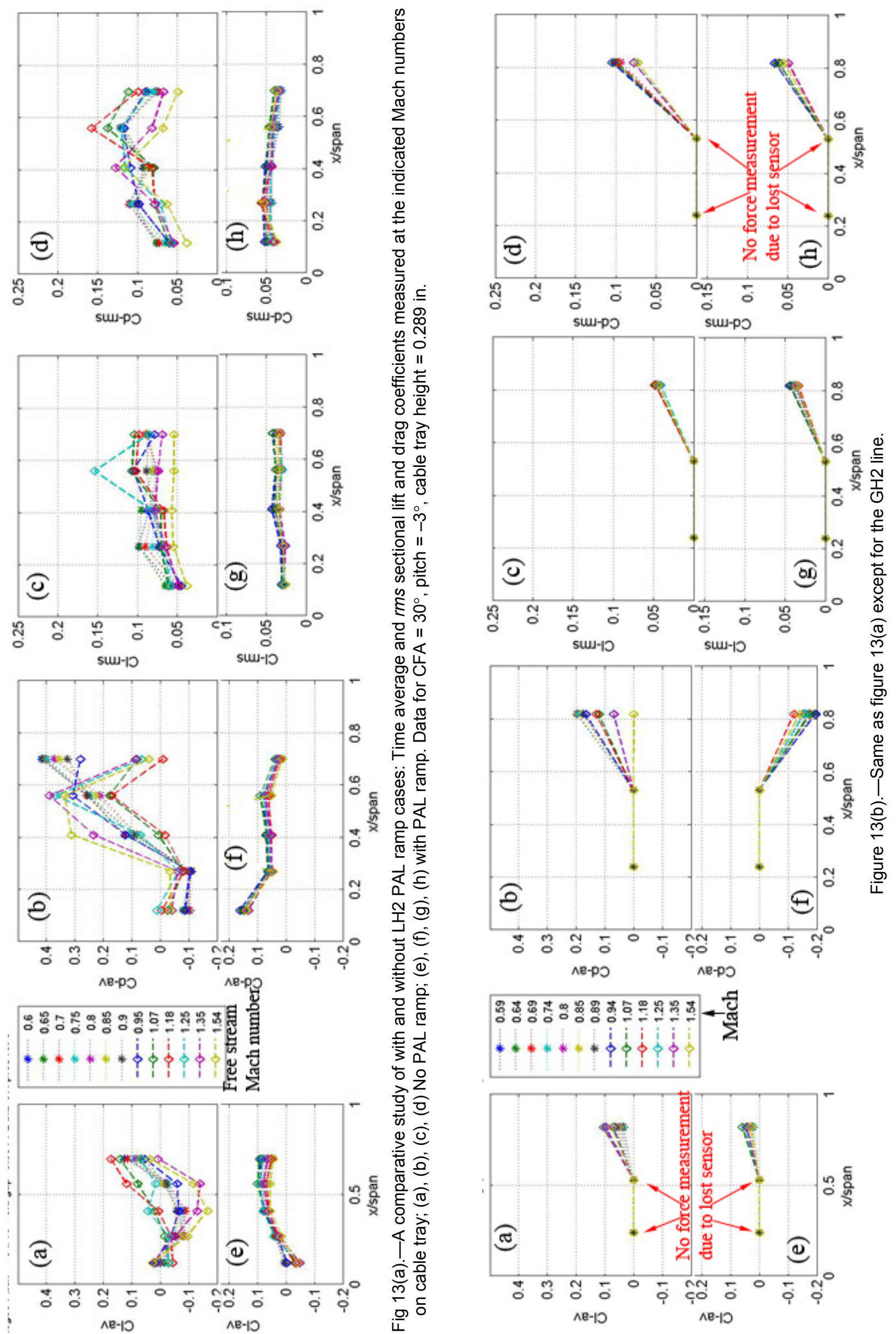

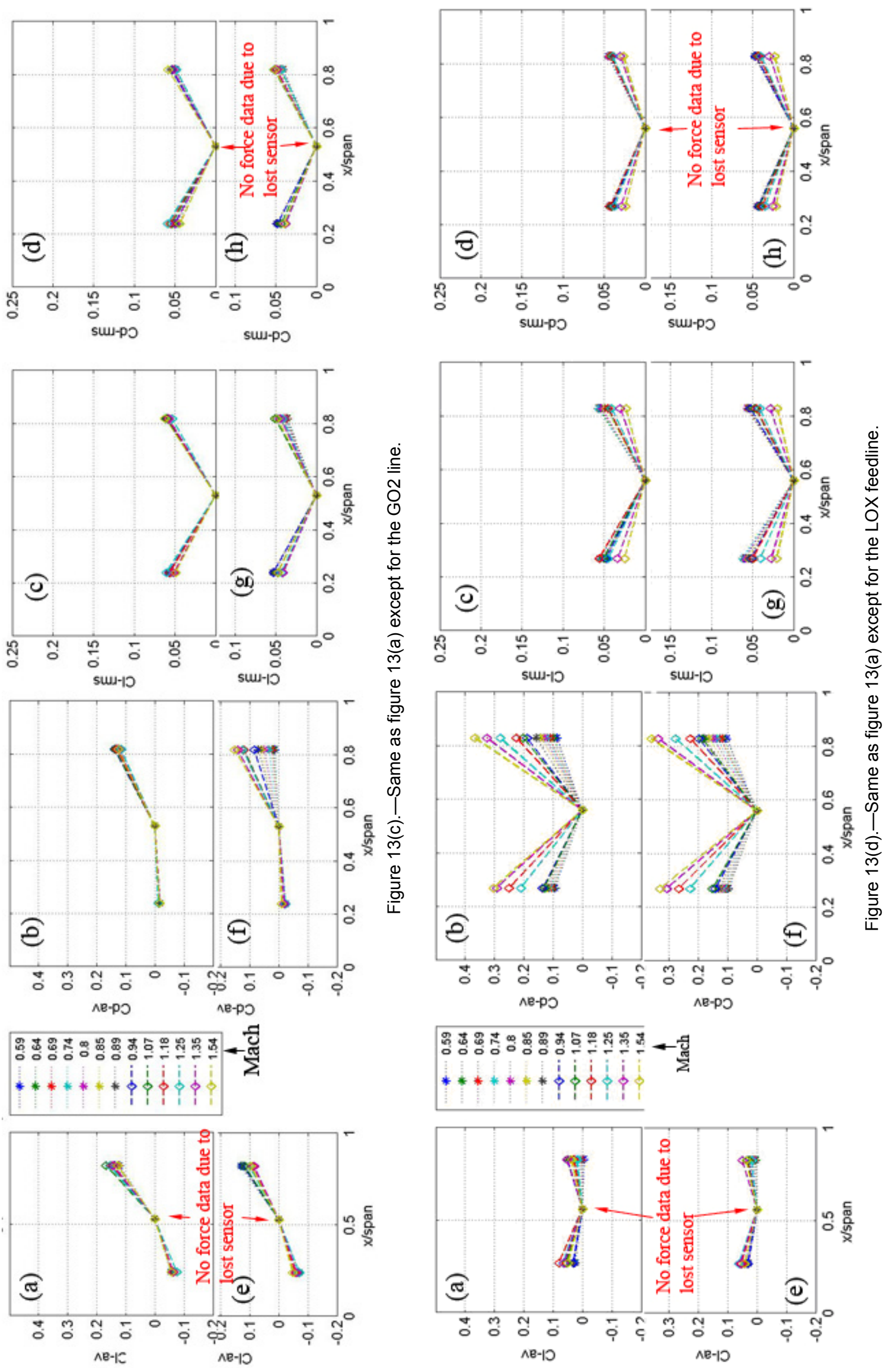


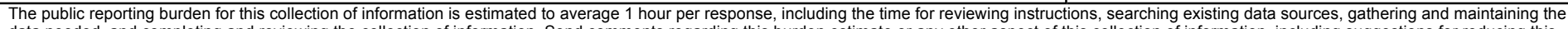

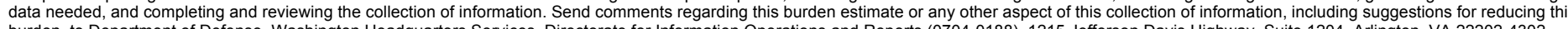

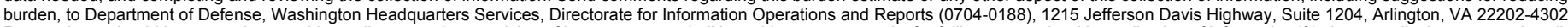

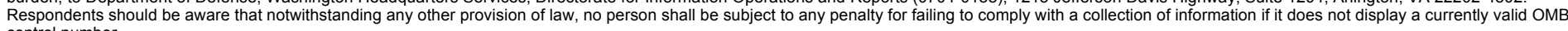

control number.
PLEASE DO NOT RETURN YOUR FORM TO THE ABOVE ADDRESS.
1. REPORT DATE (DD-MM-YYYY)
2. REPORT TYPE
3. DATES COVERED (From - To)

01-04-2008

Technical Memorandum

\section{TITLE AND SUBTITLE}

Estimation of the Unsteady Aerodynamic Load on Space Shuttle External Tank

Protuberances From a Component Wind Tunnel Test

\section{5a. CONTRACT NUMBER}

5b. GRANT NUMBER

5c. PROGRAM ELEMENT NUMBER

6. AUTHOR(S)

Panda, Jayanta; Martin, Fred, W.; Sutliff, Daniel, L.

5d. PROJECT NUMBER

5e. TASK NUMBER

5f. WORK UNIT NUMBER

WBS 510505.04.03.01

\section{PERFORMING ORGANIZATION NAME(S) AND ADDRESS(ES)}

National Aeronautics and Space Administration

8. PERFORMING ORGANIZATION REPORT NUMBER

John H. Glenn Research Center at Lewis Field

E-16380

Cleveland, Ohio 44135-3191

\section{SPONSORING/MONITORING AGENCY NAME(S) AND ADDRESS(ES)}

National Aeronautics and Space Administration

Washington, DC 20546-0001

\section{SPONSORING/MONITORS ACRONYM(S) NASA}

\section{SPONSORING/MONITORING} REPORT NUMBER

NASA/TM-2008-215155; AIAA-20080232

\section{DISTRIBUTION/AVAILABILITY STATEMENT}

Unclassified-Unlimited

Subject Category: 18

Available electronically at http://gltrs.grc.nasa.gov

This publication is available from the NASA Center for AeroSpace Information, 301-621-0390

\section{SUPPLEMENTARY NOTES}

\section{ABSTRACT}

At the wake of the Columbia (STS-107) accident it was decided to remove the Protuberance Aerodynamic Load (PAL) Ramp that was originally intended to protect various protuberances outside of the Space Shuttle External Tank from high buffet load induced by cross-flows at transonic speed. In order to establish the buffet load without the PAL ramp, a wind tunnel test was conducted where segments of the protuberances were instrumented with dynamic pressure transducers; and power-spectra of sectional lift and drag forces at various span-wise locations between two adjacent support brackets were measured under different cross flow angles, Mach number and other conditions. Additionally, frequency-dependent spatial correlations between the sectional forces were also established. The sectional forces were then adjusted by the correlation length to establish span-averaged spectra of normal and lateral forces that can be suitably "added" to various other unsteady forces encountered by the protuberance. This paper describes the methodology used for calculating the correlation-adjusted power spectrum of the buffet load. A second part of the paper describes wind-tunnel results on the difference in the buffet load on the protuberances with and without the PAL ramp. In general when the ramp height is the same as that of the protuberance height, such as that found on the liquid Oxygen part of the tank, the ramp is found to cause significant reduction of the unsteady aerodynamic load. However, on the liquid Hydrogen part of the tank, where the Oxygen feed-line is far larger in diameter than the height of the PAL ramp, little protection is found to be available to all but the Cable Tray.

\section{SUBJECT TERMS}

Space shuttle; Wind tunnel tests; Dynamic loads analysis

\begin{tabular}{|c|c|c|c|}
\hline \multicolumn{3}{|c|}{ 16. SECURITY CLASSIFICATION OF: } & \multirow{2}{*}{$\begin{array}{l}\text { 17. LIMITATION OF } \\
\text { ABSTRACT } \\
\text { UU }\end{array}$} \\
\hline $\begin{array}{l}\text { a. REPORT } \\
\text { U }\end{array}$ & $\begin{array}{l}\text { b. ABSTRACT } \\
\text { U }\end{array}$ & $\begin{array}{l}\text { c. THIS } \\
\text { PAGE } \\
\text { U }\end{array}$ & \\
\hline
\end{tabular}

18. NUMBER
OF
PAGES

24 19a. NAME OF RESPONSIBLE PERSON

STI Help Desk (email:help@sti.nasa.gov) 19b. TELEPHONE NUMBER (include area code) 301-621-0390 

